\title{
AD Digital
}

ISSN: 2697-3391

\section{Trauma craneoencefalico grave en covid-19}

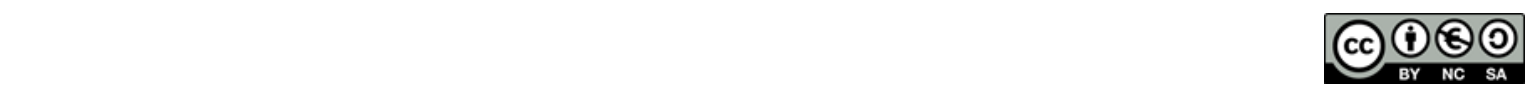

Trauma craneoencefálico grave en covid-19

María Isabel Jara Jimbo. ${ }^{1}$, Gabriela Anabel Aguilar Albito. ${ }^{2}$ \& Alfredo Daniel Pucha Landacay. ${ }^{3}$

DOI: https://doi.org/10.33262/anatomiadigital.v3i4.1410

\begin{abstract}
.
Introduction: Cranioencephalic trauma includes any physical injury or functional deterioration of the cranial content, secondary to a sudden exchange of mechanical energy. According to severity in relation to its Glasgow Scale, it is classified as Mild, Moderate and Severe. It frequently causes cerebral contusion, lacerations, direct damage to the cranial nerves and hemorrhages located at the cortical and subcortical levels, its resolution is surgical through Decompressive Craniectomy, its complications include cerebral edema, intracranial hypertension and secondary cerebral injuries of systemic cause. The onslaught of the coronavirus has taken over the world, being on January 7, 2020, when the Chinese authorities identified as the causative agent of the new outbreak of viral pneumonia a new type of virus of the
\end{abstract}

Resumen. Introducción: El trauma craneoencefálico incluye cualquier lesión física o deterioro funcional del contenido craneal, secundario a un intercambio brusco de energía mecánica. según severidad en relación a su Escala Glasgow, se clasifica en Leve, Moderado y Grave. Frecuentemente provoca contusión cerebral, laceraciones, daño directo de pares craneales y hemorragias ubicadas a nivel cortical y subcortical su resolución es quirúrgica mediante Craniectomía Descompresiva, sus complicaciones incluyen edema cerebral, hipertensión intracraneal y lesiones cerebrales secundarias de causa sistémica. La embestida del coronavirus se ha apoderado del mundo siendo el 7 de enero de 2020 cuando las autoridades chinas identificaron como agente causante del nuevo brote de neumonía viral a un nuevo tipo de virus de la familia Coronaviridae que

\footnotetext{
${ }^{1}$ Universidad Nacional de Loja, Facultad de Medicina Humana, Carrera de Medicina, Loja, Ecuador bellisajj9@hotmail.com iD https://orcid.org/0000-0003-2931-4989

${ }^{2}$ Universidad Nacional de Loja, Facultad de Medicina Humana, Carrera de Medicina, Loja, Ecuador gabbyaguilar094@gmail.com (iD https://orcid.org/0000-0002-4346-1742

${ }^{3}$ Universidad Nacional de Loja, Facultad de Medicina Humana, Carrera de Medicina, Loja, Ecuador aldaniellp11922@hotmail.com (iD) https://orcid.org/0000-0002-9506-7966
} 
Coronaviridae family that has subsequently been called SARS-CoV2-2019 . Objective: To socialize the different clinical manifestations and complications that occur in patients with TBI associated with Covid19. Results: Cranioencephalic Trauma requires the immediate detection and correction of secondary complications of systemic origin that aggravate the primary injury in which currently it is necessary to rule out covid-19 since the uncertainty of the diagnosis of Covid-19 that is associated with another pathology could lead To an erroneous count of COVID-19 deaths, especially because it presents nonspecific symptoms, the different prognostic scales to predict its severity are moderately suggestive that they can guide our treatment, but they do not present good sensitivity and their use is controversial as well. Established drug therapy holds great promise when applied early in the course of the disease, but its utility in advanced stages may be questionable. It can occur in any individual without a common base pattern, so we cannot rule it out in any patient who comes to our emergency service since it can be linked and aggravate any clinical or surgical disease as exhibited in the present case. Conclusiones: In TBI, immediate access to brain tomography is vital for immediate and timely management, since it depends on the access time to surgical resolution, which impacts the patient's prognosis. Despite the pandemic plus the demand for patients, it has been shown that serious head trauma can be adequately managed as long as coordination is maintained with the pre and intrahospital health system. posteriormente ha sido denominado SARSCoV2-2019. Objetivo: Socializar las diferentes manifestaciones clínicas y complicaciones que se presentan en pacientes con TCE asociado a Covid-19. Resultados: El Trauma Craneoencefálico requiere inmediatamente detectar y corregir complicaciones secundarias de origen sistémico que agravan la lesión primaria en las que en la actualidad se requiere descartar covid-19 ya que la incertidumbre del diagnóstico de Covid-19 que se coliga a otra patología podría llevar a un recuento erróneo de las muertes de COVID-19, especialmente por que presenta clínica inespecífica, las diferentes escalas pronosticas para predecir la severidad del mismo son medianamente sugestivas que pueden guiar nuestro tratamiento, pero no presentan buena sensibilidad y su uso es controversial así mismo la farmacoterapia establecida es muy prometedora cuando se aplica temprano en el curso de la enfermedad, pero su utilidad en etapas avanzadas puede ser dudosa. Se puede presentar en cualquier individuo sin un patrón de base en común, por lo que no podemos descartarlo en ningún paciente que llegue a nuestro servicio de emergencia ya que puede ligarse y agravar cualquier enfermedad clínica o quirúrgica como se exhibe en el presente caso. Conclusiones: En el TCE el acceso inmediato a tomografía de encéfalo es vital para el manejo inmediato y oportuno pues depende del tiempo de acceso a la resolución quirúrgica lo que impacta al pronóstico del paciente. A pesar de la pandemia más la demanda de pacientes se demuestra que se puede manejar adecuadamente traumas craneoencefálicos graves siempre y cuando se mantenga una 
Vol. 3, N4, p. 6-23, octubre-diciembre, 2020

coordinación con el sistema de salud pre e intrahospitalario.

Keywords: Trauma, cranioencephalic, coronavirus, SARS-CoV2-2019

Palabras claves: Trauma, craneoencefálico, coronavirus, SARS-CoV2-2019

\section{Introducción.}

El Trauma Craneoencefálico se define como una lesión física o deterioro funcional de contenido craneal secundario a un intercambio brusco de energía mecánica. Esta definición incluye conmoción, contusión, hemorragia o laceración del cerebro, cerebelo y tallo encefálico. (Rozman, 2016).

Su clasificación según severidad, Glasgow, Leve, Moderado y Grave, y en relación al daño causado: Lesiones primarias; causadas por la fuerza involucrada en el trauma que se subclasifican en, Lesiones primarias focales; localizadas en una región específica, por ejemplo: contusión cerebral, laceraciones, daño directo de pares craneales y hemorragias ubicadas a nivel cortical y subcortical, Lesiones primarias difusas; que ocurren por el impacto del trauma, mecanismos de aceleración desaceleración, conmoción cerebral y al daño axonal difuso. Lesiones secundarias; injuria primaria, origen intracraneal o extracraneal (Cruz 2019).

Actualmente se clasifican topográficamente las lesiones asociadas al TCE, con la escala de Marshall (Charry, 2019) (Ver figura N0 1)

Figura $\mathbf{N}^{\circ} 1$ Clasificación de Marshall para TCE basada en hallazgos tomográficos a la llegada del paciente a urgencias

\begin{tabular}{|c|c|c|c|c|c|}
\hline \multicolumn{6}{|c|}{ CLASIFICACION TOMOGRAFICA DE MARSHALL O DEL TRAUMATIC COMA DATA BANK } \\
\hline CATEGORIA & DEFINICION & CISTERNAS & DLM & $\begin{array}{l}\text { LESION } \\
\text { DE TIPO } \\
\text { MASA }\end{array}$ & MORTALIDAD \\
\hline $\begin{array}{l}\text { LESION } \\
\text { DIFUSA I }\end{array}$ & Patología intracraneal no visible & Normales & Ninguna & Ninguna & $9,6 \%$ \\
\hline $\begin{array}{c}\text { LESION } \\
\text { DIFUSA II }\end{array}$ & $\begin{array}{c}\text { Cisternas presentes con desviación } \\
\text { de líneas media } 0-5 \mathrm{~m} Y / \mathrm{O} \text { : presencia } \\
\text { de lesiones densas de densidad alta } \\
\text { o mixta no mayor a } 25 \mathrm{cc} \text {. Puede } \\
\text { incluir fragmentos óseos o cuerpos } \\
\text { extraños. }\end{array}$ & Presentes & $0-5 \mathrm{~mm}$ & $\begin{array}{c}\text { Ninguna } \\
>25 \mathrm{cc}\end{array}$ & $13,5 \%$ \\
\hline $\begin{array}{l}\text { LESION } \\
\text { DIFUSA III } \\
\text { (EDEMA) }\end{array}$ & $\begin{array}{l}\text { Cisternas comprimidas o ausentes } \\
\text { con desviación de línea media entre } \\
0-5 \mathrm{~mm} \text {. No hay lesiones altas o } \\
\text { mista de densidad mayores de } 25 \mathrm{cc} \text {. }\end{array}$ & $\begin{array}{c}\text { Comprimidas } \\
\text { o Ausentes }\end{array}$ & $0-5 \mathrm{~mm}$ & $\begin{array}{c}\text { Ninguna } \\
>25 \mathrm{cc}\end{array}$ & $34,0 \%$ \\
\hline $\begin{array}{c}\text { LESION } \\
\text { DIFUSA IV }\end{array}$ & $\begin{array}{l}\text { Desviación de línea media entre } \\
>5 \mathrm{~mm} \text {. Lesiones de densidad alta o } \\
\text { mixta no mayores a } 25 \mathrm{cc} .\end{array}$ & $\begin{array}{c}\text { Comprimidas } \\
\text { o Ausentes }\end{array}$ & $>5 \mathrm{~mm}$ & $\begin{array}{c}\text { Ninguna } \\
>25 \mathrm{cc}\end{array}$ & $56,2 \%$ \\
\hline
\end{tabular}




\begin{tabular}{|c|c|c|c|}
\hline $\begin{array}{c}\text { MASA } \\
\text { EVACUADA }\end{array}$ & $\begin{array}{c}\text { Cualquier lesión quirúrgicamente } \\
\text { evacuada }\end{array}$ & $\begin{array}{l}\text { Ninguna } \\
>25 \mathrm{cc}\end{array}$ & $38,8 \%$ \\
\hline $\begin{array}{c}\text { LESION } \\
\text { TIPO MASA } \\
\text { NO } \\
\text { EVACUADA }\end{array}$ & $\begin{array}{l}\text { Lesión de densidad alta o mixta } \\
\text { mayor a } 25 \text { cc no evacuada. }\end{array}$ & $\begin{array}{l}\text { Masa }> \\
25 \mathrm{cc}\end{array}$ & $52,8 \%$ \\
\hline
\end{tabular}

Fuente: Revista Chilena de Neurocirugía 43: 177-182, 2017

La exploración neurológica en un paciente que ha sufrido TCE debe ser minuciosa, el examen neurológico debe practicarse a intervalos regulares y se tiene que incluir siempre el examen de pupilas y la valoración del nivel de conciencia. Sus manifestaciones clínicas están íntimamente relacionadas con las estructuras cerebrales afectadas en el traumatismo, puede existir alteraciones del estado de conciencia, signos neurológicos y alteraciones de las funciones vitales, los signos de alarma son: focalidad neurológica, cefalea progresiva, vomito en proyectil, agitación psicomotora y convulsiones. (Ortiz, 2010.)

Su Fisiopatología regida por la Doctrina Monro-Kellie, predomina la valoración de presión intracraneal la cual está mediada por tres elementos: el parénquima cerebral que ocupa $80 \%$ del espacio intracraneal, el líquido cefalorraquídeo (LCR) y el volumen sanguíneo cerebral (VSC), con un $10 \%$ cada uno; y se determina por el resultado de la suma de las presiones ejercidas por los 3 compartimentos dentro del cráneo. La doctrina Monro-Kellie describe los principios que guían la homeostasis de presión intracraneal normal. La hipotesis establece que "si el cráneo está intacto, entonces la suma de los volúmenes del cerebro, el líquido cefalorraquídeo (LCR) y el volumen de sangre intracraneal es constante. (Brown,2016)

Las lesiones más frecuentes son: Fracturas de la base de cráneo que representa el 4\% de los TCE y asocian un $25 \%$ a fractura del macizo facial o lesiones cervicales, fosa anterior el signo del panda o mapache (equimosis periorbitaria), rinorragia, ceguera y anosmia. En fosa media: hemotímpano, otorragia, otorraquia, hipoacusia, estrabismo y parálisis facial periférica. En la fosa posterior: el signo de Battle que es hematoma en la apófisis mastoide y lesión del ix al xii pares craneales, Hematoma epidural: representa el $47 \%$ de lesiones y su fuente del sangradoe es en un $85 \%$ de origen arterial (la arteria meníngea media es la fuente más común de los HEDs de la fosa media). Hematoma subdural: es más frecuente que el epidural y se le asocia hasta en $30 \%$ de los casos severos, es secundario a la ruptura de las venas puente entre la corteza cerebral y senos venosos. Los datos de mal pronóstico son desviación de la línea media, mayor tamaño de la colección de sangre, contusión cerebral y lesiones extracraneanas. Hemorragia Subaracnoidea: hemorragia que ocurre en el espacio subaracnoideo y la cisterna basal, es uno de los factores para un mal pronóstico, pues incrementa dos veces el riesgo de mortalidad.23 Las manifestaciones clínicas de la hemorragia subaracnoidea incluyen cefalea severa (78\%), deterioro de conciencia (68\%), vómito $(48 \%)$ y crisis convulsivas $(7 \%)$. (Perez,2020) 


\section{ADDigital}

ISSN: 2697-3391

Vol. 3, N4, p. 6-23, octubre-diciembre, 2020

Lesiones menos frecuentes: Hematomas Intraparenquimatosos: secundarios a una contusión cerebral con ruptura de vasos sanguíneos. Generalmente ocurren en el lóbulo frontal y temporal, pero pueden ocurrir en otras áreas del cerebro como el cerebelo o el tallo. Se pueden presentar otros datos como alteraciones en el patrón respiratorio o hipotensión, aunque este último puede no ser consecuencia del TCE. La Lesión Axonal Difusa. Su localización es más común en la región frontal y temporal. Es causante de los comas de más de seis horas de evolución, En las lesiones difusas se incluyen las alteraciones axonales, vasculares, hipóxicas y la congestión cerebral. Corresponde al 3.3\% de los TCE y la mortalidad global es de alrededor del 50\% (Conolly,2010).

Su tratamiento se basa específicamente en la resolución quirúrgica como la Craniectomía Descompresiva: que se define como el retiro de un gran fragmento óseo de la bóveda craneal, para incrementar el espacio de acomodación cerebral dentro de su continente. Se han descrito tres tipos de abordajes para la descompresión intracraneana. El abordaje frontal unilateral o bilateral, el abordaje temporal unilateral o bilateral y el abordaje frontotemporoparietal que es el más frecuente más durotomía y duroplastía, se realiza en forma estrellada, se drenan colecciones o hematomas en caso de ser necesario y se procede a duroplastía evitando que quede tensa y que esto vaya a limitar la expansión del tejido encefálico. También se ha descrito la posibilidad de dejar la duramadre abierta, sin realizar durorrafia o duroplastía. (Starke,2017).

Al aumentar la PIC sobre el valor normal (20 mmHG), el tejido cerebral puede llevar a herniaciones, lo que resulta en discapacidad o muerte. Es entonces cuando la craniectomía descompresiva podría ser una opción en el manejo de estos pacientes. En la actualidad hay pocos estudios prospectivos, randomizados que recomiendan la $\mathrm{CD}$ en el TCE, se describen los estudios más mencionados en la literatura mundial. (Brown, 2016)

La craniectomía descompresiva temprana. La CD temprana es aquella que se realiza en las primeras 24 horas, para el manejo de la HIC refractaria. Con respecto al mejor momento para realizar una CD, existen dos estudios: Akyuz en el 2010 y Wen en el 2011,. Ambos compararon la CD temprana versus la CD tardía (realizada después de 24 horas del trauma de cráneo). Akyus, hizo un estudio observacional con 76 pacientes, en donde 40 pacientes se operaron de forma temprana, encontrando que aquellos que fueron sometidos de forma temprana a CD resultaron en un mejor pronóstico (GOS 4-5 el 50\% de los operados tempranamente versus un $27,8 \%$ de los operados tardíamente, $\mathrm{p}=0,0018$ ) (3). Wen realiza un estudio prospectivo con 44 pacientes, de los cuales 25 se operaron de forma temprana y cuyos criterios de inclusión fueron que el paciente no hubiera recibido terapia de hiperventilación, tratamiento con barbitúricos o drenaje previo de LCR. La diferencia en pronóstico y mortalidad en ambos grupos fue muy similar. Dada la discrepancia entre estos dos estudios y la carencia de otros estudios estadísticamente significativos, con respecto al tiempo óptimo de realizar la cirugía, es que las últimas guías de la Fundación de Trauma 


\section{ADDigital}

ISSN: 2697-3391

www.anatomiadigital.org

Vol. 3, N4, p. 6-23, octubre-diciembre, 2020

Cerebral, no incluyen recomendación alguna acerca del mejor momento para realizar la descompresión intracraneana. (NEJM,2016)

Las principales complicaciones en el TCE incluyen edema cerebral, hipertensión intracraneal y lesiones cerebrales secundarias de causa sistémica. El edema cerebral se define como el aumento del parénquima cerebral a expensas de líquido intersticial o intracelular, produciendo un aumento de la PIC por efecto de masa. (López, 2019).

La hipertensión intracraneal es un estado patológico que se caracteriza por una adición de volumen a la bóveda intracraneal. La presión intracraneal normal varía entre 3-15 mmHg. La manifestación clínica de la elevación de la pic más consistente es un descenso en el nivel de conciencia, lo que indica reducción del flujo sanguíneo cerebral. (Cruz, 2019).

La embestida del coronavirus 2 asociado al síndrome respiratorio agudo severo el (COVID19) se ha apoderado del mundo en una pandemia y ha desafiado la cultura, la economía y la infraestructura sanitaria de su población. Se ha vuelto cada vez más importante que los sistemas de salud y sus médicos adopten un marco consolidado universal para reconocer la progresión por etapas de la enfermedad COVID-19 la asociación de esta patología a toda enfermedad que pueda presentarse en un paciente sintomático o asintomático para desplegar e investigar la terapia dirigida que probablemente salve vidas. (Intramed,2020)

El 31 de diciembre de 2019, la Comisión Municipal de Salud y Sanidad de Wuhan (provincia de Hubei, China) informó sobre un grupo de 27 casos de neumonía de etiología desconocida, con una exposición común a un mercado mayorista de marisco, pescado y animales vivos en la ciudad de Wuhan, incluyendo siete casos graves. El inicio de los síntomas del primer caso fue el 8 de diciembre de 2019. El 7 de enero de 2020, las autoridades chinas identificaron como agente causante del brote un nuevo tipo de virus de la familia Coronaviridae que posteriormente ha sido denominado SARS-CoV-2, cuya secuencia genética fue compartida por las autoridades chinas el 12 de enero. El periodo de incubación medio es de 5-6 días, con un rango de 0 a 24 días (OMS 2020). El intervalo serial medio calculado en China con los primeros 425 casos fue 7,5 días con una desviación estándar de $\pm 3,4$ días (IC 95\% ). El tiempo medio desde el inicio de los síntomas hasta la recuperación es de 2 semanas cuando la enfermedad ha sido leve y 3-6 semanas cuando ha sido grave o crítica. El tiempo entre el inicio de síntomas hasta la instauración de síntomas graves como la hipoxemia es de 1 semana, y de 2-8 semanas hasta que se produce la muerte (OMS, 2020).

La actual pandemia provocada por el coronavirus COVID-19 representa un buen ejemplo de infección vírica asociada a una respuesta inflamatoria sistémica y activación de la coagulación en los pacientes sintomáticos. las infecciones virales inducen una respuesta inflamatoria sistémica acompañada de una «tormenta de citocinas», que provoca una alteración del balance entre los mecanismos pro- y anticoagulantes y ello favorece la disfunción endotelial, elevación del factor von Willebrand y de factor tisular, promoviendo 


\section{ADDigital}

ISSN: 2697-3391

www.anatomiadigital.org

Vol. 3, N4, p. 6-23, octubre-diciembre, 2020

activación de los mecanismos de coagulación. Las alteraciones de coagulación y complicaciones trombóticas son frecuentes en estos pacientes. En una serie retrospectiva que incluyó 183 pacientes reportaron que un 71,4\% de los que fallecieron cumplían criterios de CID, frente al 0,6\% de los supervivientes. (OPS,2020)

Se han descrito alteraciones de las pruebas de coagulación incluyendo aumento del dímero D (36-50\%), prolongación del tiempo de protrombina (30\%) y tromboplastina parcial activado (TTPA) (16\%) y trombocitopenia (20-30\%). Los pacientes infectados por este virus además de desarrollar CID pueden presentar trombosis venosas y/o embolismo pulmonar, así como arteriales, habiéndose descrito episodios de isquemia en los dedos de las extremidades inferiores que pueden ocasionar gangrena. Resultados muy recientes obtenidos a partir de pacientes en el área de Wuhan en China han demostrado que el dímero D, un marcador de generación de trombina y de fibrinólisis, constituye un índice pronóstico relevante de mortalidad. Dichos estudios indican que niveles de dímero D superiores a $1000 \mathrm{ng} / \mathrm{mL}$ se asocian con un riesgo 18 veces superior de mortalidad, hasta el punto de que en la actualidad se incluyen en el screening de todo paciente sintomático COVID-19 positivo. El hecho de que una coagulopatía esté presente en estos pacientes ha promovido que se planteen estrategias antitrombóticas, sobre todo en los pacientes que ingresan en la UCI y/o muestran daño orgánico o episodios isquémicos como el descrito previamente; por ello, logramos comprender que la mala ventilación e hipoxemia persistente, aumentan la mortalidad. (OPS, 2020)

Los criterios de sospecha clínica de covid-19 se asocian con enfermedad respiratoria aguda (fiebre y al menos un signo/ síntoma de enfermedad respiratoria: tos, dificultad para respirar), e historial de viaje o residencia a un país epidemiológico o una localidad del Ecuador que informa transmisión comunitaria de COVID-19, durante 14 días antes del inicio de los síntomas; Paciente con enfermedad respiratoria aguda y contacto con un caso COVID-19 confirmado o probable (ver definición de contacto cercano) en los 14 días previos al inicio de los síntomas. (Jaen,2020)

Los criterios de riesgo para covid-19 en el Ecuador, se ha establecido a pacientes desde los 50 años (más de 60 años en datos internacionales). Mujeres embarazadas, Inmunocomprometidos, Comorbilidades: Hipertensión, obesidad, asma grave, enfermedad pulmonar obstructiva crónica, fibrosis pulmonar, enfermedad renal crónica, diabetes, enfermedad cardiovascular, cáncer, tabaquismo y enfermedad cerebrovascular, Signos de Choque, SatO2 menor del 90\%, Hemoptisis, 2 criterios de qSOFA, Evidencia de trastorno de la coagulación. (IESS, 2020)

Se los clasifica en paciente de manejo ambulatorio y otro grupo amerita hospitalización, se inicia medidas de soporte Tromboprofilaxis, Antibióticos en caso de sobreinfección, seguimiento con: Saturación < $90 \%$ a Aire Ambiente o soporte de oxígeno, control de Pa02/Fi02 < 300 (Quito: < 210), Radiografía con infiltrados bilaterales, PCR: 10 veces valor 


\section{AD Digital}

ISSN: 2697-3391

normal, Ferritina: $1.000 \mathrm{ng} / \mathrm{ml}$, Dímero D: 10 veces del valor normal, LDH: 2 veces valor normal. Ingreso a UTI, se considera cuando existe diagnóstico de COVID-19 severo definido por (uno o más): Disnea, Frecuencia respiratoria $\geq 30$ / min, Hipoxemia: $\mathrm{PaO} 2<$ al 75mmHg o SatO2 < 93\% a nivel del mar; en altura $\mathrm{PaO} 2<60 \mathrm{mmHg}$ o SatO2 < 90\%,PaO2/FiO2: < 300 , Infiltrados pulmonares > 50\% campo pulmonar en 24 a 48 horas en radiografía, tomografía y/o ecografía. (IESS, 2020)

Figura No 2 Consenso multidisciplinario informado en la evidencia sobre el tratamiento de covid-19 en hospitalización

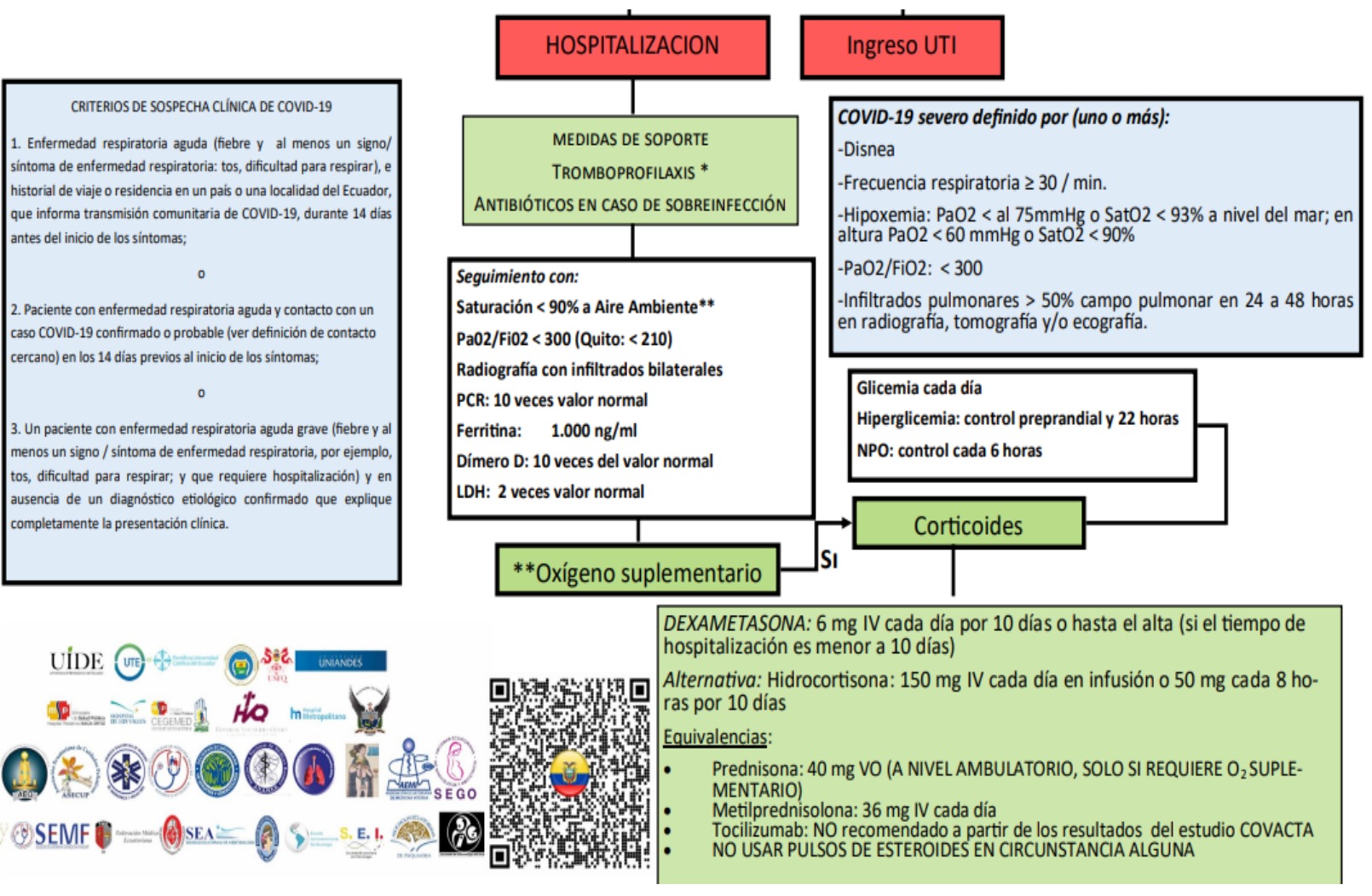

Fuente: Página oficial del Ministerio de Salud Publica

Su Tratamiento involucra; Corticoides: Al inicio de la pandemia existía publicaciones sobre contraindicaciones. Su importancia inicia a mediados de abril cuando estudios hablaron sobre disminución de mortalidad, En abril Critical Care (2020) fue un ensayo clínico aleatorio 244 pacientes críticos usaron una dosis equivalente a hidrocortisona $200 \mathrm{mg} / \mathrm{día}$, buscaron Reducir la mortalidad a 28 días, pero no se describió claramente la población en estudio; Cruz (2020), cohorte retrospectivo, 463 pacientes, uso metilprednisolona $1 \mathrm{mg} / \mathrm{kg} / \mathrm{día}$, con reducción de mortalidad 13,9 vs $23,9 \%$ redujo mortalidad a los 28 días, pero las complicaciones superaron a los beneficios; Zhikage ye, (2020) en 330 pacientes críticos, uso de corticoides, pero aumento mortalidad; Fadel (2020) uso en 213 pacientes metilprednisolona $0.5-1 \mathrm{mg} / \mathrm{kg} /$ día x 3 días, se redujo días de hospitalización, pero no 


\section{ADDigital}

ISSN: 2697-3391

www.anatomiadigital.org

Vol. 3, N4, p. 6-23, octubre-diciembre, 2020

disminuye mortalidad. Existen guías de práctica clínica: Surviving sepsis campaing. Intensive care med(2020), en relación a paciente Covid 19 y uso de corticoide en pacientes con hipoxemia, se indica metilprednisolona 0.5 y $1 \mathrm{mg} / \mathrm{kg}$ x día por 7 días, pero se encontró aumento en relación a pacientes con sobre infección bacteriana más micosis invasivas que reingresaban. RECOVERY, es un ensayo clínico aleatorio, que busco disminuir mortalidad a los 28 días, la población utilizada fue 2104 pacientes, informo que, el uso de Dexametasona $6 \mathrm{mg}$ cada 24 horas en terapia intensiva demostró beneficios en población que recibió oxigeno ( Covid 19 moderado y severa); Es decir, que no se recomienda uso en pacientes si apoyo de oxígeno ni severidad leve por no encontrar beneficio. (Cared,2020)

Anticoagulación: A nivel pulmonar, la importancia radica por la formación de microtrombos, problemas como liberación de fibrina, elevación de Dinero D, con un inicio temprano de tendrá un impacto en mortalidad. Se recomienda uso de heparina de bajo peso molecular en enfermedad severa siempre con Dimero D mayor de 3 o elevación de fibrinógeno; además la trombocitopenia es un factor de mal pronóstico de evolución y no se recomienda el uso de antiplaquetarios en este grupo de pacientes. Pero uso de profilaxis en pacientes con antecedentes previos, factores de riesgo moderado y severo de trombo embolia pulmonar, disminuye el riesgo de mortalidad a los 28 días. Se debe mantener la anticoagulación previa, interacciones médicas, la duración de anticoagulación en pacientes con antecedentes de TEP / trombosis asociado a catéter, se debe mantener 6 semanas a 3meses, para evitar muerte súbita post alta. Estudio de Mucha (2020) analizo valores de Dimero D para manejo de anticoagulación, encontró que con valores menores a $3.0 \mathrm{ug} / \mathrm{ml}$, se debe administrar profilaxis con enoxaparina subcutánea, siempre valorando injuria renal y demostró que con valores mayores a $3 \mathrm{ug} / \mathrm{ml}$, se recomienda seguimiento con ecografía para inicio de dosis de trombo profilaxis, heparinas intravenosa, o enoxaparina subcutánea.(Mucha,2020)

Los antibióticos carecen de efectos antiviral, deben administrarse únicamente en casos de coinfección bacteriana, especialmente en pacientes con neumonía comunitaria viral por SARS-Cov-2 que requiere hospitalización; Sospecha de coinfección bacteriana en presencia de leucocitosis, procalcitonina mayor a $1.50 \mathrm{ng} / \mathrm{ml}$, radiografía de tórax con infiltrados predominantemente unilaterales ; Se recomienda no prescribir cefalosporinas de tercera generación por la alta tasa de resistencia bacteriana reportada en nuestro país. Presentamos Algoritmo de manejo sospecha de infección por sars-cov2 o covid-19 confirmado. (Acuña,2020) (Ver Figura No 2).

Según el Consenso multidisciplinario informado en la evidencia sobre tratamiento Covid 19, evidencia otras Recomendaciones que no se utilizaron en el presente caso pero que se encuentran enfrentando distintas controversias por lo que se creyó necesario mencionar como lo son; Ivermectina, cloroquina, hidroxicloroquina, plasma convaleciente, rendesivir, tocilizumab. 


\section{AD Digital}

ISSN: 2697-3391

\section{Presentación de Caso.}

Se trata de paciente masculino de 26 años de edad de estado civil soltero de profesión policía sin antecedentes de importancia recibido en la sala de urgencias del Hospital IESS Loja, porque hace 9 horas previo su ingreso es víctima de accidente de tránsito en calidad de conductor de motocicleta, no se conoce detalles específicos de cinemática del accidente, posterior al mismo presenta deterioro inmediato y progresivo del nivel de conciencia por lo que personal paramédico procede a intubación endotraqueal, hasta ser trasportado a esta casa de salud en código rojo, a su ingreso en emergencia paciente orointubado, recibe presión positiva con sistema bbm, con collar cervical y férula espinal, presencia de sonda nasogastrica, se evidencia equimosis palpebral izquierda con presencia de laceración a nivel de región fronto parietal izquierda, cuello no valorable por presencia de collar cervical, tórax campos pulmonares estertores crepitantes bibasales, disminución de murmullo vesicular a nivel de base izquierda, corazón ruidos cardiacos de tono e intensidad normofonetico, no ruidos sobreañadidos, sincrónicos con el pulso, abdomen blando depresible no doloroso a la palpación RHA disminuidos. Exámenes de Laboratorio. (Ver tabla $\mathrm{N}^{\mathrm{o}} 1$ )

Se realiza tac simple de tórax y de cráneo, en la toografía de encéfalo se evidencia presencia de hematoma subdural agudo frontotemporo parietal de lado izquierdo.(Ver imagen $\mathrm{N}^{\mathrm{o}} 1$ )

Tabla $\mathbf{N}^{\circ} 1$ Tac simple de tórax y de cráneo

\begin{tabular}{|c|c|c|c|c|c|c|c|c|}
\hline EXAMEN & DIA 1 & DIA 7 & DIA 10 & DIA 20 & DIA 25 & DIA 30 & DIA 40 & DIA 45 \\
\hline PCR & 23,57 & 225,5 & 149 & 136,6 & 123 & 192 & 96 & 12 \\
\hline LEUCOCITOS & 23,5 & 13,5 & 10,8 & 11 & 8,5 & 13,4 & 33,6 & 8,3 \\
\hline NEUTROS & 22,83 & 11,4 & 7,68 & 8,03 & 7,7 & 11,14 & 27,5 & 5,21 \\
\hline LINFOCITOS & 1,5 & 900 & 1,55 & 3,01 & 1,69 & 1,51 & 0,65 & 2,29 \\
\hline HB & 15,67 & 11,5 & 10,5 & 8,9 & 9,3 & 10,5 & 15 & 11,8 \\
\hline Нто & 47,5 & 32,7 & 31,1 & 27,6 & 27,3 & 31,1 & 49 & 36,5 \\
\hline PLAQUETAS & 223 & 300 & 371 & 506 & 600 & 602 & 430 & 320 \\
\hline LDH & 396 & 500 & 1000 & 573 & 400 & 347 & 270 & 300 \\
\hline FERRITINA & 160,7 & 329 & 400 & 500 & 250 & 282 & 270 & 261 \\
\hline DIMERO D & 7,19 & 4,2 & 3,17 & 2,5 & & 1,5 & & 2,76 \\
\hline UREA & 18 & 20 & 22 & 19 & 50 & 91 & 65 & 11 \\
\hline CREATININA & 0,87 & 0,77 & 0,7 & 0,65 & 1,3 & 3,9 & 1,9 & 1,2 \\
\hline GLUCOSA & 73 & & & & & & & \\
\hline AST & 48 & & & 50 & & 80 & & 55 \\
\hline ALT & 36 & & & 40 & & 44 & & 88 \\
\hline TP & 13 & & 14 & & & & & \\
\hline TTP & 28,9 & & 31 & & & & & \\
\hline INR & 120 & & & & & & & 0,1 \\
\hline PROCALCITONINA & 0,49 & & & & & 0,01 & & \\
\hline
\end{tabular}




\section{ADDigigital}

ISSN: 2697-3391

Vol. 3, $\mathrm{N}^{\circ} 4$, p. 6-23, octubre-diciembre, 2020

\begin{tabular}{|c|c|c|c|c|c|c|c|c|}
\hline NA/K & $134 / 4,7$ & & & & & & & \\
\hline GSA: PH & 7,36 & 7,4 & 7,36 & 7,4 & 7,42 & 7,39 & 7,33 & 7,49 \\
\hline $\mathrm{PCO} 2$ & 29 & 30 & 33 & 31,7 & 31,2 & 32 & 31,3 & 45 \\
\hline PO2 & 170 & 80 & 73 & 40 & 135 & 45 & 55,6 & 70 \\
\hline $\mathrm{HCO} 3$ & 16,3 & 16 & 18,6 & 20 & 20 & 19 & 16 & 22 \\
\hline TROPONINA & 46,4 & & & & & & & \\
\hline \multicolumn{9}{|l|}{ COLESTEROL } \\
\hline TSH & 2,4 & & & & & & & \\
\hline RTPCR-SARSCOV2 & & $(+)$ & & & & & $(-)$ & \\
\hline $\begin{array}{c}\text { PRUEBA RAPIDA- } \\
\text { IGM-IGG } \\
\text { HEMOCULTIVO }\end{array}$ & $\begin{array}{l}\text { IGM +, } \\
\text { IGG - } \\
(-)\end{array}$ & & $\begin{array}{c}\text { IGM +, } \\
\text { IGG- }\end{array}$ & & $\begin{array}{l}\text { IGM+, } \\
\text { IGG+ }\end{array}$ & & & $\begin{array}{l}\text { IGM- } \\
\text { IGG- }\end{array}$ \\
\hline H.RECTAL & $(-)$ & & & & & & & \\
\hline
\end{tabular}

Elaborado por : Grupo de investigación.

Imagen $\mathbf{N}^{\mathbf{1} 1}$ Tac simple de tórax y de cráneo

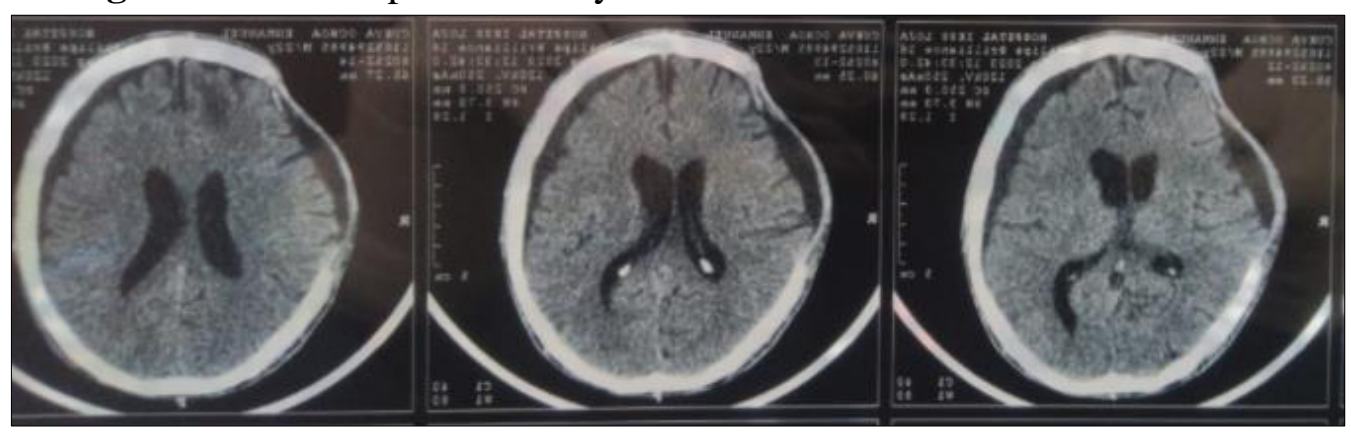

Fuente: As400 IESS-Loja.

En tórax presencia de imágenes en vidrio deslustrado subpleurales con áreas de consolidación a nivel de ambas bases pulmonares. (Ver Imagen $\mathrm{N}^{\circ} .2 \mathrm{~A}$ y $2 \mathrm{~B}$ )

\section{Imagen $\mathbf{N}^{\circ} .2$ En tórax}

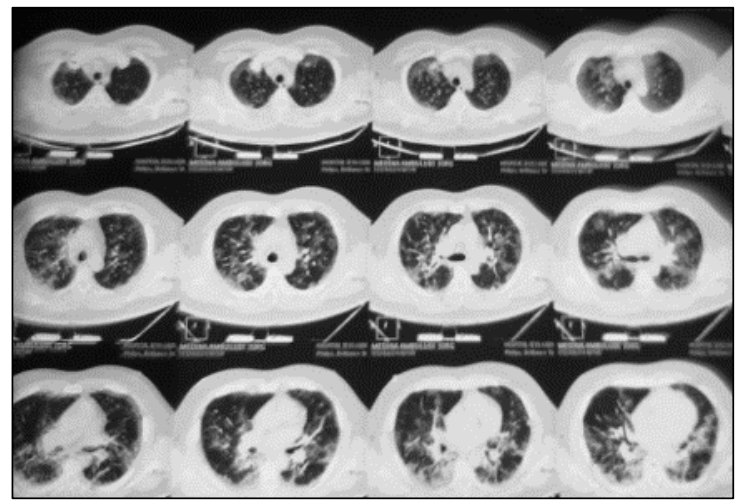

A

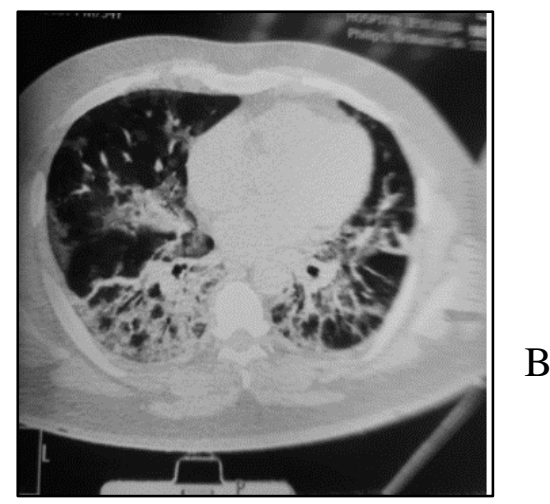

Elaborado por : Grupo de investigación. 


\section{AD Digitital}

ISSN: 2697-3391

En el ECOFAST en área no se observa presencia de líquido a nivel de cavidad abdominal, no se evidencia presencia de líquido pericárdico. Paciente en condición crítica con pronóstico reservado es ingresado a nuestra unidad de cuidados intensivos.

Paciente que permanece 45 días en cuidados intensivos ingresado por trauma craneocefalico grave, concomitantemente presenta neumonía por covid-19, durante su estancia en UCI con apoyo ventilatorio invasivo, sin apoyo vasopresor, se mantiene estable hemodinamicamente luego de ser intervenido por neurocirugía para craneotomía descompresiva frontotemporal derecha más evacuación de hematoma subdural agudo frontotemporal derecho además colocación de catéter intracraneal (medición de presión intracraneal, recibió cuidados de neuromonitoreo con sensor de PIC y mantuvo controles de PIC entre 9 -11, posteriormente se decide realizar vacación durante 12 horas de sedación para valorar respuesta neurológica, asiste a la ventilación mecánica, sin respuesta a la estimulación dolorosa, se decide reiniciar sedación por asincronía ventilatoria. se retira sensor de PIC por riesgo de neuro infección sin complicación alguna, se decide realizar traqueostomía por intubación prolongada sin ninguna complicación con posterior acople ventilatorio, realiza episodios convulsivos por lo que inicia anticomicial, paciente permanece estacionario por varios días se logra destete progresivo y prolongado de sedoanalgesia pero permanece con estado neurológico secuelar con episodios de agitación psicomotriz, no conexiones con el medio, no obedece órdenes y no responde al dolor, a las $72 \mathrm{~h}$ paciente logra autonomía ventilatoria por lo que se logra destete ventilatorio completo al momento con apoyo de oxígeno por mascarilla con buena tolerancia, hemodinamicamente estable y con buena tolerancia a dieta, con micción y deposición espontanea por lo que se decide pase a piso de clínica covid.

En su recuperación en el piso de clínica durante su hospitalización se mantiene con buena mecánica ventilatoria que permite progresar su parte respiratoria y manejar oxígeno a bajos flujos, sin embargo paciente se muestra comprometido neurológicamente ya que paciente presenta un Glasgow 10/15, ( O:4 V:3 M:3).

Se mantiene con sonda nasogástrica y sonda vesical durante 5 días posteriormente se somete a gastrostomía programada, sin complicaciones, se logra destete de oxígeno a bajo flujo aportado por puntas nasales.

Recibió tratamiento a base ceftriaxona 2 gramos iv cada 12 horas 10/10, piperacilina mas tazobactam 4.5 gramos intravenoso cada 6 horas d7, fenitoína 125 miligramos intravenoso cada 8 horas 30/30, levetiracetam 1 gramo intravenoso cada 8 horas 20/20, modafinilo (alertex) $200 \mathrm{mg}$ por sng cada 12h, enoxaparina 20mg sc qd 15/15, metilprednisolona 125 mgiligramos intravenoso cada día día 3/3 ( 16/06/2020). Se recibe fisioterapia, se educa a los familiares manejo de paciente y es dado de alta. 


\section{AD Digitital}

ISSN: 2697-3391

www.anatomiadigital.org

Vol. 3, N4, p. 6-23, octubre-diciembre, 2020

\section{Discusión.}

En enfoque de nuestro caso clínico podemos deducir que los traumatismos causados por el tránsito constituyen un impacto muy grave en cuanto a los problemas de salud no solamente pública si no de desarrollo, es una de las causas en las que muchos de los pacientes llegan a perder la vida casi 1,2 millones de personas cada año y resultan heridas o quedan discapacitadas entre 20 millones y 50 millones. (OMS- Banco Mundial ,2016). En cuanto a la edad de nuestro paciente destacamos que por lo general en las personas menores de 35 años es la primera causa de muerte y discapacidad (F. Alberdi, 2017). Actualmente en el trauma craneoencefálico la tasa de mortalidad está reflejada en 579 por 100.000 persona/año, principalmente debido a caídas y/o accidentes vehiculares y esta puede estar asociada al sexo (predominantemente en hombres), edad y/o país (más alta en países en vía de desarrollo) (Herrera, 2018).

En Ecuador ocupa la cuarta causa de muerte según datos proporcionados por el Instituto Nacional de Estadística y Censos (INEC) y primera de discapacidad entre los sujetos menores de 45 años con importantes consecuencias en el ámbito personal, familiar y social (Mendaro, 2017), nuestro paciente entra en un rango etario concordante con este dato nacional y en un grupo de cuidado por su grado severo de discapacidad como dependiente incluso para sus tareas habituales, consideramos el gran imparto no solo personal sino familiar y social que ha generado su traumatismo hoy con una crisis a nivel mundial generada por la pandemia COVID 19.

El traumatismo craneoencefálico grave por tránsito (TCEG), se asocia con frecuencia a lesiones traumáticas y no traumáticas del sistema respiratorio, que provocan una reducción de la presión arterial de oxígeno $(\mathrm{PaO} 2)$ y retención de dióxido de carbono, con necesidad de ventilación artificial mecánica (VAM) para su corrección en la mayoría de los casos, la VAM permite utilizar estrategias terapéuticas como la hiperventilación moderada y transitoria, para lograr valores de presión arterial de dióxido de carbono (PaCO2) entre 30-35mmHg, que asociado mejoran parámetros como $\mathrm{PaO} 2$, regula vasoconstricción cerebral, presión intracraneal (PIC), permitiendo un adecuado flujo sanguíneo cerebral (FSC) . (Lacerda, 2020). Lo que representa maniobras de primera línea para el control de la hipertensión intracraneal que se instauraron en nuestro paciente adecuadamente, además del acceso temprano a craneotomía descompresiva a pesar del momento en que nos encontramos por la pandemia.

El síndrome de distrés respiratorio (SDRA) es una condición amenazante para la vida, cuando el TCE es concomitante con SDRA, el manejo ventilatorio se hace complejo y difícil debido a que los objetivos ventilatorios entran en conflicto entre sí. El manejo para este tipo de pacientes es prevenir las lesiones secundarias a la hipoxia, manteniendo una adecuada perfusión cerebral y óptima oxigenación de las estructuras intracraneales; he aquí la 


\section{ADDigital}

ISSN: 2697-3391

importancia de la ventilación mecánica la cual se vuelve requisito en el manejo de pacientes con TCE severo. (Arrieta, 2018). Nuestro paciente debuta con cuadro de SDRA debido a su TECG, formo parte del $16 \%$ de los pacientes que lo presentan durante la VMA sin embargo rescatamos el trabajo multidisciplinario y de terapia intensiva al no entra en la tasa de mortalidad, ya que el egresa vivo, pero con un grado severo de discapacidad en su alta nos mantenemos expectantes de su pronóstico a revaloración dentro de 6 meses.

En el trauma craneoencefálico, el 25\% se acompaña de síndrome de distrés respiratorio agudo, que se suele asociar a reacción inflamatoria sistémica, y lleva al sistema respiratorio a una reducción de la tolerancia al estrés y adicionalmente a un posible aumento de las complicaciones pulmonares asociadas a la ventilación (VILI). Nuestro paciente llego a un Hospital de Segundo Nivel de la red de Salud Pública, y a pesar del momento se pudo concretar tratamientos para mejorar su pronóstico ya que la ventilación de Alta Frecuencia Oscilatoria ha demostrado mejoría en la oxigenación al momento de enfrentarse a un SDRA, también comparativamente, tiene una menor incidencia de VILI respecto al manejo ventilatorio mecánico convencional en los valores de $\mathrm{PaCO} 2$ entre 31 y $35 \mathrm{mmhg}$, valor que impacta en vasodilatación cerebral y modificación de la hemodinámica cerebral empeorando el pronóstico. (Arrieta, 2018),

Los accidentes cerebrovasculares y las lesiones cerebrales traumáticas tienen una alta prevalencia en todo el mundo y siguen siendo motivos comunes de visitas al servicio de urgencias (CDC, 2020).

Los pacientes mayores de 65 años son una cohorte creciente, para niveles lesiónales similares tienen el doble de mortalidad que los jóvenes, debido a su elevada comorbilidad y los tratamientos asociados" (Saavedra, 2020), siendo el mismo otro punto en nuestro paciente su edad en 22 años y la no asociación a enfermedades de base. En Ecuador en el 2018 en el Hospital Luis Vernaza se estudiaron un total de 96 pacientes con diagnóstico de TCE grave, con una Me de edad de 32,50 (RI 23,00 - 43,00).

El 28,1\% (27/96) de los pacientes fueron mujeres y el 71,9\% (69/96) varones. La mediana de días de hospitalización en UCI fue de 9,00 (RI 6,00 - 15,00) días, en complicaciones se describen Neurológicas: 32,3\% (31/96) Respiratorias: 32,3 (31/96) Cardiovasculares: 8,3\% (8) Sepsis: 19,8\% (19/96) Renal: 6,2\% (6/96) Otros: 1\% (1/96),y se determinó que la mortalidad en el Hospital Luis Vernaza, en la UCI, los pacientes con TEC grave fue del 29.2\%. (López 2018).

Otras de las manifestaciones reportadas son los trastornos motores, los cuales a su vez requieren de un preciso manejo por parte del equipo de trabajo, con vistas a erradicarlo o limitar su afectación (Mendaro, Caracterización epidemiológica y neurológica del traumatismo craneoencefálico frontal durante cinco años en Villa Clara. Medicentro, 2017). 


\section{ADDigital}

ISSN: 2697-3391

Vol. 3, N4, p. 6-23, octubre-diciembre, 2020

Desde el punto de vista imagenológico, las lesiones predominantes fueron las fracturas lineales, seguida de las contusiones sin efecto de masa y los hematomas subdurales, siendo la TAC, la técnica imagenológica de elección para el diagnóstico, pronóstico, control evolutivo de las lesiones iniciales del TCE y de la respuesta a la terapia. De esta forma, algunas guías afirman que dicha herramienta debe ser realizada en aquellos pacientes con trauma moderado o severo, debido a la mayor incidencia de hemorragia intracraneal aguda (Benítez, 2019), nuestro paciente, por la necesidad de ventilación mecánica prolongada, uso de drogas analgésicas, paralizantes, y por complicaciones en terapia como Hiperactividad simpática paroxística, su evaluación neurológica fue muy compleja.

Cuando a lo expuesto deducimos que al recibirse a un paciente joven con trauma craneoencefálico con estado de conciencia muy deteriorado la literatura y estadísticas demuestran que este paciente tiene más del $60 \%$ de probabilidad de recuperación un $40 \%$ de riesgo de lesiones neurológicas en el SNC como; paraplejia etc. cuando se trata de un paciente con compromiso pulmonar como; fumador, obeso, entre otras patologías el riesgo de mortalidad asciende a un 50\%. En la actualidad no solo debemos buscar estos factores de riesgo sino la predominante patología que ha conmocionado al mundo el covid-19, las lesiones fibrocicatrizales a nivel pulmonar causan en un paciente oxigeno dependencia e incluso la muerte, además se busca factores como: edad más de 65 años biomarcadores; linfopenia. Ferritina, LDH, Dimero D, sin embargo según estudios realizados en (china) en pacientes jóvenes existen muy poca incidencia de severidad, correlacionándose estrechamente con nuestro paciente, la estancia hospitalaria prolongada, se relaciona con evolución estacionaria, y daño neurológico , por presiones arteriales oscilantes, y periodos prolongado de hipoxemia, Los datos de la PIC tienen gran valor pronóstico, de tal manera que la Hipertensión Intracraneal se asocia al mal pronóstico debido a la isquemia cerebral que causa, En el Hospital Luis Vernaza en el 2018, se estudió 96 pacientes un 31,3\% de los pacientes con HIC fallecieron. Un aspecto importante a señalar es que la PIC se monitoriza solo en un 44,8\% de los casos, existiendo HIC en el 68,1\% de los pacientes.

Esto nos debe obligar a reflexionar sobre el bajo porcentaje de neuromonitorizados de nuestra UCI. razón por la cual coincidimos con que nuestro paciente fue dado de alta hospitalaria luego de 60 días en hospitalización con grave secuela neurológica, considerando el arduo esfuerzo de personal médico por evitar estados de descompensación señalamos que al tratarse a su vez de un paciente con diagnostico covid - 19 denota un compromiso pulmonar vital para la recuperación de paciente ya que interfiere con el estado fisiológico de oxigenación adecuada par aun paciente resumimos que el presente caso debuto con traqueostomía sin embargo pese a la misma fue de importancia el destete completo y con autonomía ventilatoria para egreso del paciente que conto con PCR negativo, la larga estancia hospitalaria con lleva a nuestros paciente a múltiples intervenciones y procedimientos rutinarios implica el riesgo de cuadros nosocomiales. Se debe enfocar en mejorar los protocolos de atención y disponer 
de nuromonitorización continua en terapia intensiva, teniendo mayor control de que parámetros que impactan en el pronóstico de todos los pacientes.

\section{Conclusiones.}

- En el trauma cráneo encefálico el acceso inmediato a tomografía de encéfalo es vital para el manejo inmediato y oportuno pues depende del tiempo de acceso a la resolución quirúrgica lo que impacta al pronóstico del paciente.

- A pesar de la pandemia más la demanda de pacientes se demuestra que se puede manejar adecuadamente traumas craneoencefálicos graves siempre y cuando se mantenga una coordinación con el sistema de salud pre e intrahospitalario.

- Cuando en el TCE más Covid-19 existe persistencia de leucocitosis. linfopenia progresiva y eosinopenia son criterios de falla terapéutica por lo que es ideal iniciar lo antes posible cambio de terapia a tratamientos que mejoren la respuesta inmune para evitar la hiperinflamación.

\section{Referencias bibliográficas.}

Arrieta, M. C. (15 de 04 de 2018). Ventilación mecánica en el paciente con trauma cerebral. Obtenido de Ventilación mecánica en el paciente con trauma cerebral: http://www.revmie.sld.cu/index.php/mie/article/view/551/html_172

Angel. Jaen. Craneoplastia en pacientes con craniectomía descompresiva por un traumatismo craneoencefálico grave. Revista Chilena de Neurocirugía 2018: 39

Conolly, S. Fundamentals of Operative Techniques in Neurosurgery. Canadá. Segunda edición.

https://www.researchgate.net/publication/336358949_Doctrina_Monro-

Kellie_fisiologia_y_fisiopatologia_aplicada_para_el_manejo_neurocritico

CDC. (19 de 04 de 2020). Centers for Disease Control and Prevention. Emergency Department Visits. Obtenido de Centers for Disease Control and Prevention. Emergency Department Visits.: https://www.cdc.gov/nchs/fastats/emergencydepartment.htm.

Cruz López AM, Ugalde Valladolid A, Aparicio Ambriz CA, Contreras Landeros LY, Carnalla Cortes M y cols, Abordaje del traumatismo craneoencefálico Aten. Fam. 2019;26(1):28-33. http://dx.doi.org/10.22201/facmed.14058871p.2019.1.67714 // https://www.medigraphic.com/pdfs/atefam/af-2019/af191g.pdf

Brown. Decompressive craniectomy in Acute Brain Injury, Handbook of Clinical Neurology. Elsevier. 2017; 140: 299-318.

Lacerda, A. J. (20 de Julio de 2020). Presión positiva al final de la espiración, presión intracraneal y presión de perfusión cerebral en el paciente pediátrico critico con 


\section{ADDigitalal}

ISSN: 2697-3391

Vol. 3, N4, p. 6-23, octubre-diciembre, 2020

traumatismo craneoencefálico. Obtenido de Presión positiva al final de la espiración, presión intracraneal y presión de perfusión cerebral en el paciente pediátrico crítico con traumatismo craneoencefálico: https://www.researchgate.net/publication/342164667_Medicina_intensiva

Mendaro, D. d. (14 de Diciembre de 2017). Caracterización neuropsicológica de pacientes con trauma craneoencefálico frontal leve y moderado. Obtenido de Caracterización neuropsicológica de pacientes con trauma craneoencefálico frontal leve y moderado.: https://www.medigraphic.com/pdfs/medicadelcentro/mec-2017/mec172b.pdf

Ortiz. A. Traumatismo encéfalo craneano, una puesta al día. Rev. Med. Clin. Condes - 2006; 17(3): $98-105$

World health Organization. Water, sanitation, hygiene and waste management for COVID$19 \quad$ [Internet]. 2020. Disponible en: https://apps.who.int/iris/bitstream/handle/10665/331305/WHO2019-NcOVIPC_WASH-2020.1-eng.pdf?sequence $=1 \&$ is Allowed $=y$

Wuhan seafood market pneumonia virus isolate Wuhan-Hu-1, complete genome. 23 de enero de 2020 [citado 7 de febrero de 2020]; Disponible en: http://www.ncbi.nlm.nih.gov/nuccore/MN908947.3

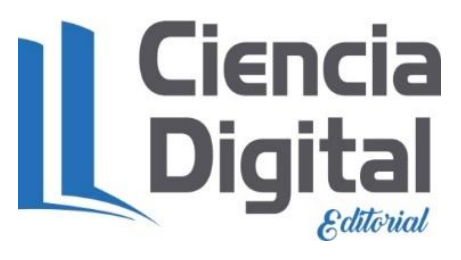




\section{PARA CITAR EL ARTÍCULO INDEXADO.}

Jara Jimbo, M. I., Aguilar Albito, G. A., \& Pucha Landacay, A. D. (2020). Trauma craneoencefalico grave en covid-19. Anatomía Digital, 3(4), 6-23. https://doi.org/10.33262/anatomiadigital.v3i4.1410

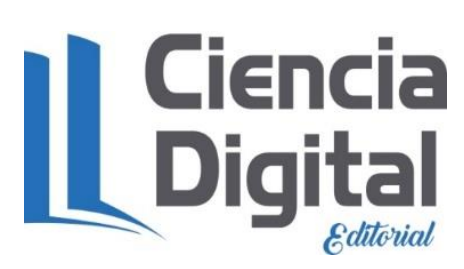

El artículo que se publica es de exclusiva responsabilidad de los autores y no necesariamente reflejan el pensamiento de la Revista Anatomía Digital.

El artículo queda en propiedad de la revista y, por tanto, su publicación parcial y/o total en otro medio tiene que ser autorizado por el director de la Revista Anatomía Digital.
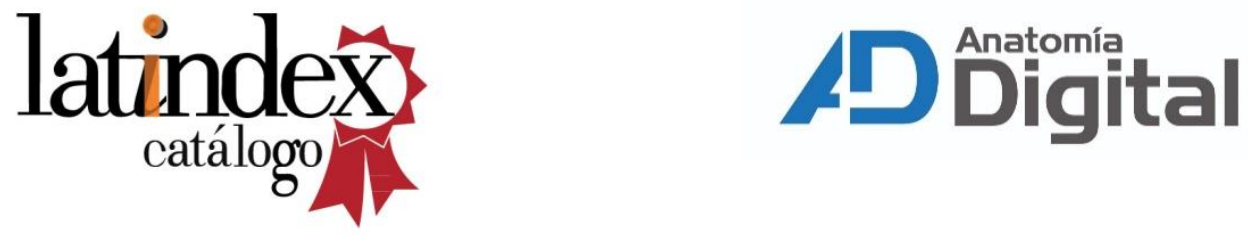